\title{
Case Report: Multiorgan Involvement with Congenital Zika Syndrome
}

\author{
Rodrigo Cachay, ${ }^{1 *}$ Alvaro Schwalb, ${ }^{1}$ Takashi Watanabe, ${ }^{1}$ Doris Guzman, ${ }^{2}$ Thomas Jaenisch, ${ }^{3,4}$ Daniel Guillén-Pinto, ${ }^{2}$ and \\ Eduardo Gotuzzo ${ }^{1}$ \\ ${ }^{1}$ Instituto de Medicina Tropical Alexander von Humboldt, Universidad Peruana Cayetano Heredia, Lima, Peru; ${ }^{2}$ Hospital Cayetano Heredia, Lima, \\ Peru; ${ }^{3}$ Heidelberg Institute of Global Health (HIGH), Heidelberg University Hospital, Heidelberg, Germany; ${ }^{4}$ Colorado School of Public Health, \\ University of Colorado, Anschutz Medical Campus, Aurora, Colorado
}

\begin{abstract}
We report the case of an infant born with congenital Zika syndrome (CZS). During the largest Zika virus (ZIKV) outbreak in Peru, the mother presented with fever and rash that were confirmed to be due to ZIKV by real-time PCR. The infant was born with severe microcephaly. Imaging revealed corpus callosum dysgenesis, lissencephaly, ventriculomegaly, and calcifications. Mild hypertrophic cardiomyopathy with diastolic dysfunction was reported in the echocardiogram. Valgus deviation of the lower extremities and a left clubfoot were diagnosed at birth. The hip ultrasound showed incipient signs of Graf type II dysplasia. The findings confirm that CZS is a multiorgan phenotype in which microcephaly is merely the tip of the iceberg. A multidisciplinary approach is needed for the evaluation of these children.
\end{abstract}

\section{INTRODUCTION}

Since its introduction in 2015, Zika virus (ZIKV) has produced multiple outbreaks in South America. Contracted through the bite of an infected mosquito or through sexual contact, ZIKV infection can impair fetal development when transmitted during pregnancy. ${ }^{1}$ Congenital Zika syndrome (CZS) is a constellation of neurodevelopmental abnormalities; however, this spectrum is not limited to the brain as it also encompasses cardiovascular compromise and bone-related disorders. $^{2,3}$ In 2019, the largest ZIKV outbreak in Peru occurred in Jaén, a high jungle city in the Cajamarca region. We report the case of an infant born with CZS with broad clinical manifestations.

\section{CASE REPORT}

A 27-year old woman presented at 10 weeks of pregnancy with a pruriginous generalized rash associated with malaise and subjective fever. She did not experience non-purulent conjunctivitis or arthralgias. She denied any past medical history or drug use. Because of the ongoing ZIKV outbreak in Jaén, a serum ZIKV real-time PCR (RT-PCR) was performed and was reported to be positive. Her symptoms resolved in a week and she was referred for obstetric follow-up.

Obstetric ultrasounds were unremarkable until the 25th week of pregnancy when findings suggested intrauterine growth restriction (IUGR). A thorough assessment at week 27 confirmed severe IUGR with an estimated fetal weight of $1,003 \mathrm{~g}(<$ first percentile), microcephaly $(<3 \mathrm{SDs})$, and lissencephaly. In addition, periventricular, basal ganglia and intraparenchymal calcifications were reported. Cardiovascular findings included right ventriculomegaly $(11 \mathrm{~mm})$ and mitral valve calcification. She had received all the recommended immunizations for pregnancy according to national guidelines. Serum IgG for rubella, Toxoplasma, and cytomegalovirus (CMV) were positive.

She delivered a baby boy at 36 weeks and 5 days gestational age. He weighed $1,900 \mathrm{~g}$ ( $<$ third percentile), was $45 \mathrm{~cm}$

\footnotetext{
*Address correspondence to Rodrigo Cachay, Instituto de Medicina Tropical Alexander von Humboldt, Universidad Peruana Cayetano Heredia, Av. Honorio Delgado 430, San Martín de Porres, Lima 15102, Peru. E-mail: rodrigo.cachay.f@upch.pe
}

long (between 10th and 50th percentile), and had a head circumference of $28 \mathrm{~cm}$ ( $<$ third percentile). He presented prominent microcephaly, retrognathia, and valgus deviation of lower extremities with a left clubfoot (Figures 1 and 2). Serum IgM for CMV and Toxoplasma were indeterminate, and IgM for herpes simplex virus 1 and 2 were negative. ZIKV testing was not performed on clinical samples collected from the neonate; likewise, genetic testing such as microarray testing or karyotyping was not performed because of testing limitations in the region.

At 2 months of age, the infant was thoroughly evaluated. $\mathrm{He}$ was irritable and appeared malnourished with weak suction. $\mathrm{He}$ had a punctiform anterior fontanelle and presented with generalized hypertony and distal quadriparesis. Deep tendon reflexes and assessment of cranial nerves were normal. Fundoscopy was unremarkable.

Head computed tomography revealed corpus callosum dysgenesis, lissencephaly, occipital horn dilatation of lateral ventricles, and calcifications of frontal white matter and basal ganglia (Figure $3 \mathrm{~A}$ and $\mathrm{B}$ ). In addition, magnetic resonance imaging showed dysmyelination and generalized pachygyria-a lissencephaly pattern compatible with neuronal migration disorders (Figure 4A-G). The electroencephalogram was abnormal, showing asymmetric, low-frequency activity predominantly on the left hemisphere without paroxysms. Evoked auditory and visual potentials were reported as normal.

A grade I/VI systolic murmur was audible over the precordium. The echocardiogram revealed mild hypertrophic cardiomyopathy with diastolic dysfunction and indirect signs of pulmonary hypertension. Lower limb radiography reported longitudinal axis deviation and bilateral internal rotation, and hip ultrasound showed incipient signs of Graf type II hip dysplasia. Physical therapy sessions were scheduled to improve tone and global developmental delay. In addition, thorough counseling on breastfeeding with formula supplementation was given.

\section{DISCUSSION}

Congenital Zika syndrome exhibits various clinical features that are a direct consequence of severe intracranial volume loss and neurological damage. ${ }^{1}$ The most notable finding of CZS is microcephaly, which has been strongly associated with infection at early gestational weeks. ${ }^{4}$ Defined as head 


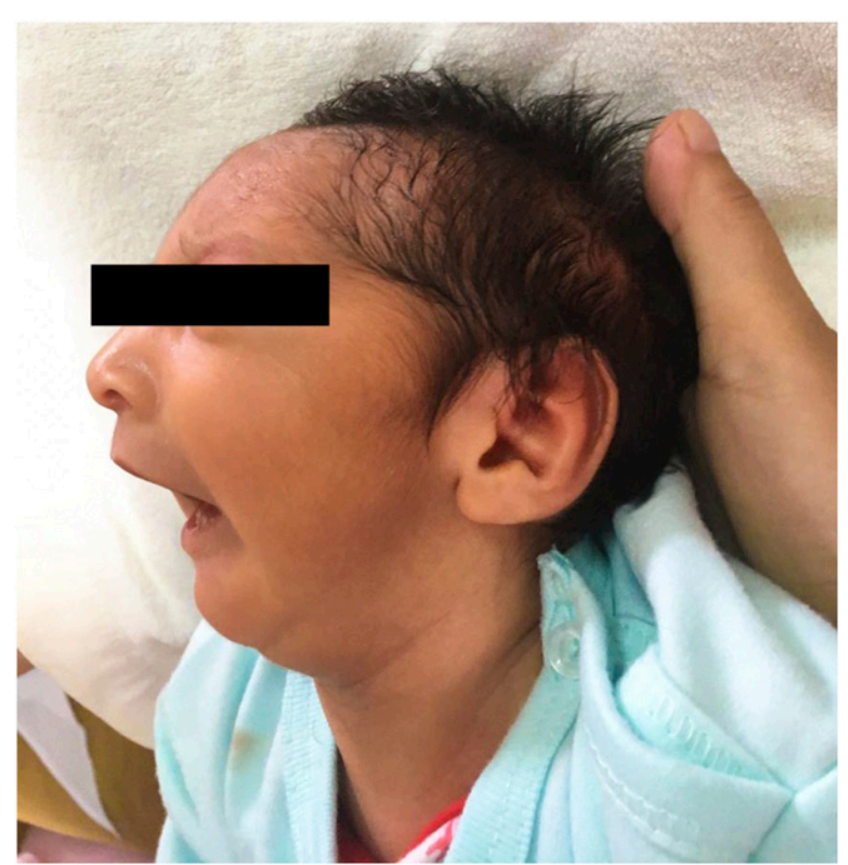

Figure 1. Prominent microcephaly and retrognathia. This figure appears in color at www.ajtmh.org.

circumference more than two SDs (or more than three SDs for severe microcephaly) below the mean or less than the third percentile for age and gender, microcephaly can be diagnosed pre- or postnatally. ${ }^{5}$ Although the rate of microcephaly in Cajamarca (the region where our patient is from) was higher than that reported in other countries before the introduction of ZIKV in South America, it is possible that this difference is explained by distinct measurement techniques or the use of standard curves in a population with smaller head circumferences. ${ }^{6}$ Nonetheless, we defined our case as CZS because of the clinical findings of the infant and the positive ZIKV RT-PCR from the mother during pregnancy.

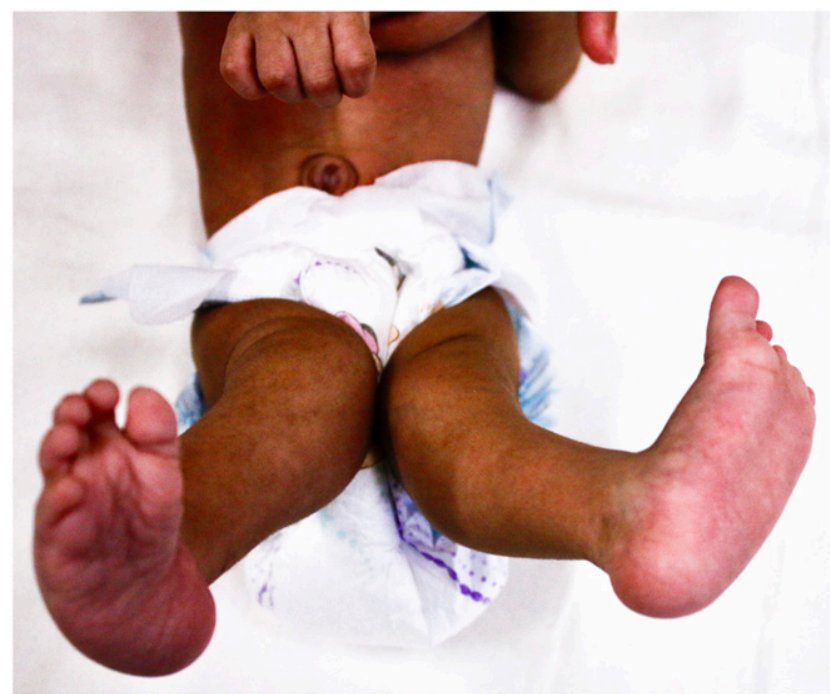

FIGURE 2. Valgus deviation of lower extremities and left talipes equinovarus. This figure appears in color at www.ajtmh.org.
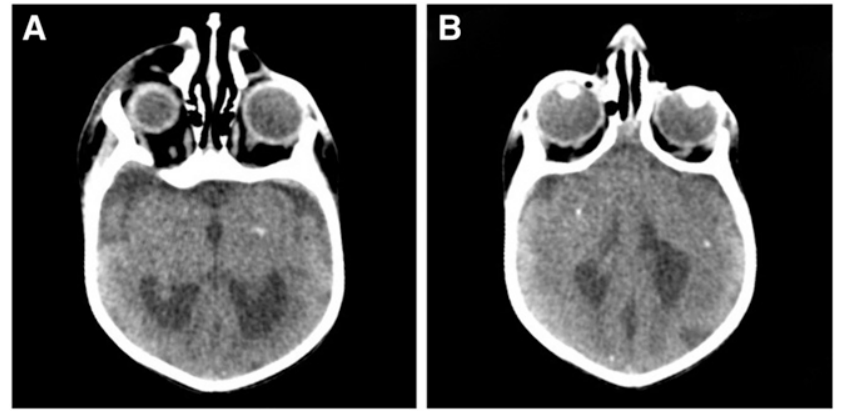

FIgURE 3. Computed tomography of the head. (A) Axial view, calcifications in the left thalamus. (B) Axial view, right occipital and bilateral temporal calcifications.

Although microcephaly in obstetric ultrasounds allows for early detection of ZIKV-related cerebral manifestations, this method should not be considered for screening of CZS as other brain findings can be seen in imaging ${ }^{7}$; these may include an underlying neuronal migration disorder due to impaired development of neuroprogenitor cells, which leads to ventriculomegaly and lissencephaly. ${ }^{8}$ Subsequent inflammation caused by the virus, manifested as intracranial calcifications predominantly at the junction between the cortex and subcortical white matter, basal ganglia, and thalamus can also be seen on ultrasound images. ${ }^{9}$ Depending on the extent of neural proliferation compromise, CZS has been found to cause sensory disabilities such as visual and hearing deficits. ${ }^{10}$ Furthermore, abnormal brain activity without epileptiform discharges has been described in $48 \%$ of children with CZS, ${ }^{11}$ although seizures have been described. ${ }^{12}$ Severe brain abnormalities impair development, and early termination of pregnancy is an option in some countries ${ }^{13}$; however, the legislation in Peru does not allow for disruption of pregnancy unless the mother's life is at risk.

In addition, cardiovascular compromise has a potential effect of ZIKV infection. Transient myocarditis, heart failure, and changes in repolarization leading to rhythm disorders, such as atrial fibrillation, non-sustained atrial tachycardia, and ventricular arrhythmia, have been reported in adult patients, ${ }^{14}$ whereas structural heart defects are a prevalent feature of in utero ZIKV exposure.,15 Echocardiographic evaluations show mild ventricular and atrial septal defects, and, as with microcephaly, the frequency of major defects was higher in infants whose mothers had ZIKV exposure early in pregnancy or who had neurological anomalies in imaging. ${ }^{2}$ Our case did not present congenital structural defects or rhythm disorders; nonetheless, pulmonary hypertension and hypertrophic cardiomyopathy were reported. Some authors suggest timely screening among CZS cases due to potential undiagnosed cardiovascular complications. ${ }^{16}$ Currently, our case does not require any medication, but further follow-up has been scheduled.

Another cell lineage to which ZIKV has demonstrated tropism is osteoblast cells by disturbing their differentiation, maturation, and function. ${ }^{3}$ The presence of ZIKV on synovial fluid correlates with the development of arthralgias in primary infection, ${ }^{17}$ but the imbalance in bone homeostasis is due to impaired osteoblast function, which can induce bone-related disorders while the fetus is developing. ${ }^{1}$ Arthrogryposis, one of the most limiting extracranial conditions, involves multiple 

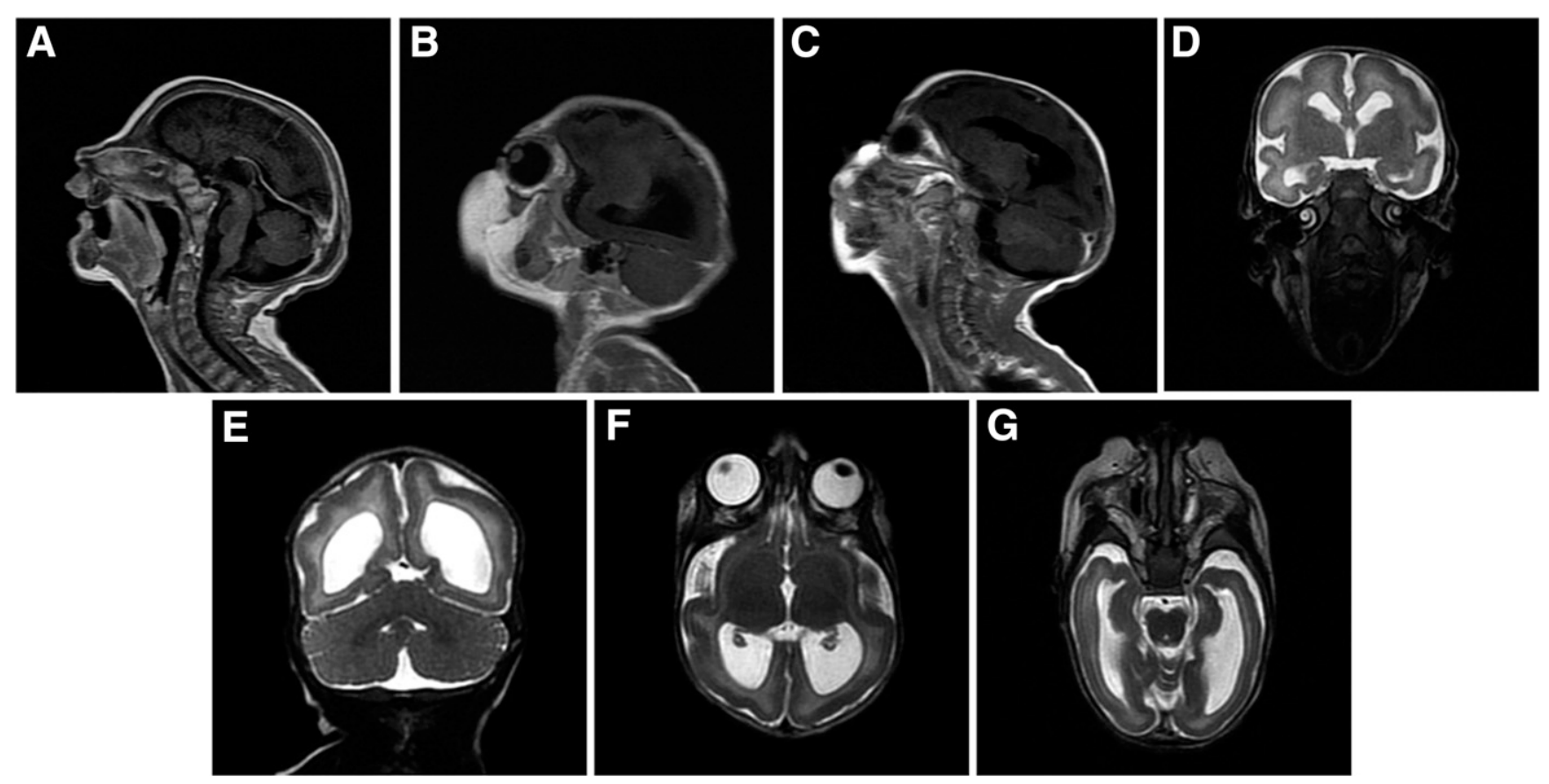

FIGURE 4. Magnetic resonance imaging of the head. (A) Sagittal view, absence of the corpus callosum and reduced brain volume at the expense of the frontal lobe. (B) Sagittal view, lissencephaly with hypoplasia of the temporal cortex. (C) Sagittal view, eyeball and optic nerve pathway. (D) Coronal view, smooth cortex with few grooves; absence of the corpus callosum without differentiation of the basal ganglia and the thalamus. (E) Coronal view, smooth cortex without cerebral grooves; evident dysmyelination. (F) Axial view, Probst bundles are seen by the occipital horns. (G) Axial view of temporal lobes with evident lissencephaly.

joint contractures, and its association with CZS has been described with flexed wrists as the most common finding. ${ }^{18}$ Other manifestations include talipes equinovarus (clubfoot), valgus deviation, and bilateral rotation of the lower limbs, and hip dislocation. ${ }^{18}$ Physical therapy is recommended to improve postural control and mobility, ${ }^{19}$ and has been suggested to improve this patient's clubfoot and angulation of the extremities.

In the short time that ZIKV has emerged as a public health emergency in South America, CSZ has presented broad clinical manifestations in vertically transmitted infants. Microcephaly is perhaps the condition with the highest negative impact on the quality of life of affected infants and their families and should be seen as the tip of the iceberg of this syndrome. Interventions based on a multidisciplinary approach should be implemented to evaluate and monitor children with CZS and reduce the burden of the disease.

Received May 6, 2020. Accepted for publication June 3, 2020.

Published online July 27, 2020.

Disclosure: The case was evaluated as part of the ZIKAlliance cohort (European Union's Horizon 2020 Research and Innovation Programme under grant agreement N. 734548) focused on the impact of ZIKV during pregnancy and the natural history of ZIKV in humans and their environment.

Authors' addresses: Rodrigo Cachay, Alvaro Schwalb, Takashi Watanabe, and Eduardo Gotuzzo, Instituto de Medicina Tropical Alexander von Humboldt, Universidad Peruana Cayetano Heredia, Lima, Peru, E-mails: rodrigo.cachay.f@upch.pe, alvaro.schwalb@ upch.pe, takashi.watanabe.t@upch.pe, and eduardo.gotuzzo@ upch.pe. Doris Guzman, Hospital Nacional Cayetano Heredia, Lima, Peru, E-mail: doris.guzman@upch.pe. Daniel Guillén-Pinto, Pediatria, Universidad Peruana Cayetano Heredia, Lima, Peru, E-mail: dguillenpinto@gmail.com. Thomas Jaenisch, Heidelberg University
Hospital, Heidelberg, Germany, and Colorado School of Public Health, University of Colorado, Anschutz Medical Campus, Aurora, CO, E-mail: thomas.jaenisch@uni-heidelberg.de.

\section{REFERENCES}

1. Moore CA et al., 2017. Characterizing the pattern of anomalies in congenital Zika syndrome for pediatric clinicians. JAMA Pediatr 171: 288-295.

2. Orofino DHG et al., 2018. Cardiac findings in infants with in utero exposure to Zika virus- a cross sectional study. PLoS NegI Trop Dis 12: e0006362.

3. Mumtaz N, Koedam M, van den Doel PB, van Leeuwen JPTM, Koopmans MPG, van der Eerden BCJ, Rockx B, 2018. Zika virus infection perturbs osteoblast function. Sci Rep 8: 16975.

4. Brady OJ et al., 2019. The association between Zika virus infection and microcephaly in Brazil 2015-2017: an observational analysis of over 4 million births. PLoS Med 16: e1002755.

5. DeSilva M et al.; Brighton Collaboration Congenital Microcephaly Working Group, 2017. Congenital microcephaly: case definition \& guidelines for data collection, analysis, and presentation of safety data after maternal immunisation. Vaccine 35: 6472-6482.

6. Santos-Antonio G, Canchihuamán F, Huamán-Espino L, Aparco JP, Pillaca J, Guillén-Pinto D, Gozzer E, 2019. Microcephaly in newborns in level II and III health facilities of the Ministry of Health of Peru [article in Spanish]. Rev Peru Med Exp Salud Publica 36: 222-230.

7. Sanz Cortes M et al., 2018. Clinical assessment and brain findings in a cohort of mothers, fetuses and infants infected with ZIKA virus. Am J Obstet Gynecol 218: 440.e1-440.e36.

8. Hussain A, Ali F, Latiwesh OB, Hussain S, 2018. A comprehensive review of the manifestations and pathogenesis of Zika virus in neonates and adults. Cureus 10: e3290.

9. de Fatima Viana Vasco Aragao M, de Lima Petribu NC, van der Linden V, Valenca MM, de Brito CAA, Parizel PM, 2019. Updated imaging findings in congenital Zika syndrome: a disease story that is still being written. Top Magn Reson Imaging 28: 1-14. 
10. Peloggia A, Ali M, Nanda K, Bahamondes L, 2018. Zika virus exposure in pregnancy and its association with newborn visual anomalies and hearing loss. Int J Gynaecol Obstet 143: 277-281.

11. Moura da Silva AA et al., 2016. Early growth and neurologic outcomes of infants with probable congenital Zika virus syndrome. Emerg Infect Dis 22: 1953-1956.

12. Pessoa A, van der Linden V, Yeargin-Allsopp M, Carvalho MDCG, Ribeiro EM, Van Naarden Braun K, Durkin MS, Pastula DM, Moore JT, Moore CA, 2018. Motor abnormalities and epilepsy in infants and children with evidence of congenital Zika virus infection. Pediatrics 141 (Suppl 2): S167-S179.

13. Carabali M, Austin N, King NB, Kaufman JS, 2018. The Zika epidemic and abortion in Latin America: a scoping review. Glob Health Res Policy 3: 15.

14. Minhas AM, Nayab A, lyer S, Narmeen M, Fatima K, Khan MS, Constantin J, 2017. Association of Zika virus with myocarditis, heart failure, and arrhythmias: a literature review. Cureus 9: e1399.

15. Cavalcanti DD, Alves LV, Furtado GJ, Santos CC, Feitosa FG, Ribeiro MC, Menge P, Lira IM, Alves JG, 2017. Echocardiographic findings in infants with presumed congenital Zika syndrome: retrospective case series study. PLoS One 12: e0175065.

16. Krittanawong $\mathrm{C}$, Zhang $\mathrm{H}$, Sun $\mathrm{T}$, 2016. Cardiovascular complications after Zika virus infection. Int J Cardiol 221: 859.

17. Colavita F, Musumeci G, Caglioti C, 2018. Human osteoblast-like cells are permissive for Zika virus replication. J Rheumatol 45: 443.

18. Serpa SC, de Melo ACMG, Gomes Lins O, van der Linden V, Leite Rolim Filho E, Oliveira Dos Santos AC, 2019. Orthopedic findings in arthrogryposis and congenital Zika syndrome: a case series. Birth Defects Res 112: 385-392.

19. Amundsen KR, Evensen KAl, 2020. Physical therapy intervention for a child with congenital Zika virus syndrome: a case report. Child Neurol Open 7: 2329048X19896190. 